\section{Intervelações entre SHBG e Esteróides Sexuais com Medidas Antropométricas, Pressão Arterial e Lipideos em Mulheres Com e Sem Diabetes Mellitus Tipo 2}

\section{RESUMO}

Com o objetivo de analisar as concentrações plasmáticas de SHBG, dos esteróides sexuais, perfil glicídico, lipídico e suas relações com variáveis clínicas, demográficas e medidas antropométricas, estudamos um grupo de 80 mulheres na pós-menopausa, 40 com diabetes mellitus (DM) tipo 2, com idade de $64,9 \pm 7,1$ anos e duração conhecida do diabetes de $13,4 \pm 1,4$ anos e 40 não diabéticas com idade de $61 \pm 8,9$ anos. Foram analisados: idade, indice de massa corporal (IMC), relação cinturaquadril (RCQ), cintura, pressão arterial sistólica (PAs) e diastólica (PAd). As mulheres diabéticas apresentaram maior freqüência de distribuição andróide de gordura (75\% vs. $50 \%, p=0,03)$, maior PAs $(p=0,01)$, maior testosterona total $(p=0,003)$, índice de testosterona livre $(p=0,002)$ e indice de resistência insulínica $(I R \mid)(p=0,000)$ do que as mulheres não diabéticas. As não diabéticas com distribuição andróide de gordura apresentaram menores niveis de SHBG do que aquelas com distribuição ginecóide $(p=0,008)$. No grupo com $I M C \geq 30 \mathrm{~kg} / \mathrm{m} 2$ e no grupo com cintura $\geq 88 \mathrm{~cm}$, as mulheres diabéticas apresentaram maior testosterona total e índice de testosterona livre do que as mulheres não diabéticas. As não diabéticas com distribuição andróide de gordura apresentaram maior indice de testosterona livre do que aquelas com distribuição ginecóide $(p=0,01)$. As diabéticas com distribuição andróide apresentaram maiores niveis de estradiol do que aquelas com distribuição ginecóide $(p=0,02)$. Em conclusão, mulheres diabéticas apresentaram maior freqüência de distribuição abdominal de gordura, estando associada à maiores concentrações de testosterona total, índice de testosterona livre e estradiol e menores concentrações de SHBG. Estes dados sugerem que o hiperandrogenismo com diminuição de SHBG, possam ser indicadores da síndrome de resistência insulínica, e poderiam, de alguma forma, agravar o grau de resistência nestes pacientes. (Arq Bras Endocrinol Metab 2000;44/3: 239-47)

Unitermos: SHBG; Esteróides sexuais; Diabetes tipo 2.

\section{artigo original}

\author{
Luciana Babia \\ Trude Dimetz \\ Helena Gazolla \\ Eliete Clemente \\ Marilia B. Gomes
}

Departamento de Medicina Interna, Disciplina de Diabetes, Unipersidade do Estado do Rio de Janeiro (UERJ), Rio de Janeiro, $R J$.

\title{
ABSTRACT
}

In order to investigate the relationship between plasma levels of SHBG. sex steroids and glucose metabolism, lipid profile, anthropometric measurements, clinical and demographic variables, we studied a group of 80 post-menopausal women, 40 with type 2 diabetes (DM2) aged 64.9 7.7.1 and with known DM duration of $13.4 \pm 1.4$ years and 40 non-diabetic aged 61 \pm 8 .9. We analyzed: age, body mass index (BMI), waist to hip ratio (WHR), waist, systolic (sBP) and diastolic blood pressure (dBP). Diabetic women had higher frequency of android adiposity ( $75 \%$ vs. $50 \%, p=0.03$ ), higher $\mathrm{sBP}(p=0.01)$, higher total testosterone $(p=0.003)$, free testosterone index $(p=0.02)$ and insulin resistance index $(I R \mid)(p=0.000)$ than non-diabetic women. Non-diabetics with android adiposity had lower levels of SHBG than those with ginecoid adiposity $(p=0.008)$. In the group with $\mathrm{BMl} \geq 30 \mathrm{~kg} / \mathrm{m} 2$ and in the group with waist $\geq 88 \mathrm{~cm}$ diabetic women had higher total testosterone and free testosterone index than non-dia- 
betic women. Non-diabetics with android adiposity had higher free testosterone index than those with ginecoid adiposity $(p=0.01)$. Diabetic women with android adiposity had higher estradiol than those with ginecoid adiposity $(p=0.02)$. In conclusion, diabetic women had higher frequency of android adiposity, associated with high total testosterone, free testosterone index and estradiol and low levels of SHBG. These results suggest that hyperandrogenism with low SHBG could be indicators of the insulin resistance syndrome, and could somehow increase the insulin resistance in these subjects. (Arq Bras Endocrinol Metab 2000;44/3: 239-47)

Keywords: SHBG; Sex steroids; Type 2 diabetes.

$\mathrm{O}$ S NÍVEIS DOS ESTERÓIDES SEXUAIS, assim como da sua principal proteína carreadora ( $\mathrm{SHBG}$ ), variam em função de fatores como sexo, idade, excesso de peso e distribuição abdominal de gordura, presença de diabetes (DM) e outras doenças crônicas.

A SHBG funciona como moduladora da oferta tissular destes hormônios. É produzida pelo fígado e sofre influências dos níveis dos esteróides sexuais (1), da insulina (2) e, possivelmente, em menor intensidade, do GH/IGF-1 (3), dos hormônios tireoideanos (1) e da prolactina (4). Parece que a insulina seria o principal hormônio regulador das concentrações circulantes de $\mathrm{SHBG}$, com vários estudos demonstrando correlação inversa entre os níveis de insulina e $\mathrm{SHBG}$, sugerindo um efeito inibitório sobre a secreção hepática (5-7). Em condições de hiperinsulinemia ocorre diminuição dos níveis de SHBG, sendo esta diminuição considerada um possível marcador de resistência insulínica e de desenvolvimento futuro de DM tipo $2 \mathrm{em}$ homens e mulheres (8-11).

Os esteróides sexuais, por mecanismos ainda não completamente esclarecidos, são capazes de influenciar o metabolismo glicídico, lipídico e a distribuição regional de gordura. Em mulheres, o aumento dos níveis de testosterona se relaciona com intolerância à glicose e hiperinsulinemia (12). Mulheres diabéticas freqüentemente apresentam menores níveis de $S H B G$ e maiores níveis de testosterona livre, refletindo um estado de hiperandrogenismo que se correlaciona com a hiperinsulinemia e distribuição abdominal de gordura (13). Nos homens, os níveis de testosterona correlacionam-se negativamente com a insulina $(14,15) \mathrm{e}$ a administração de testosterona não parece deteriorar a tolerância à glicose, sendo capaz até de melhorar a sensibilidade insulínica (16). Observa-se, então, efeitos paradoxais dos androgênios nos sexos feminino e mas- culino. Parece haver uma pequena faixa de variação na concentração circulante de testosterona na qual a ação da insulina é ótima, sendo que níveis muito elevados ou muito baixos reduzem a sensibilidade à insulina em homens e mulheres $(17,18)$.

Com o objetivo de analisar as concentrações plasmáticas de SHBG, dos esteróides sexuais, perfil glicídico, lipídico e suas relações com variáveis clínicas, demográficas e medidas antropométricas, estudamos um grupo mulheres com DM2 regularmente atendidas no ambulatório do Hospital Universitário Pedro Ernesto e um grupo de mulheres não diabéticas.

\section{PACIENTES E MÉTODOS}

Foram estudadas 80 mulheres, sendo 40 mulheres não diabéticas com idade de $61 \pm 8,9$ anos e 40 com DM2 com idade de $64,9 \pm 7,1$ anos, classificados de acordo com critérios clínicos e metabólicos descritos no National Diabetes Data Group (19). O grupo diabético apresentava duração conhecida do diabetes de $13,4 \pm 1,4$ anos (0-35). As demais características dos grupos estão descritas na tabela 1 . Os tipos de tratamento utilizados para o DM foram os seguintes: dieta: $\mathrm{n}=2(5 \%)$, sulfoniluréias: $\mathrm{n}=12(30 \%)$, metformina: $\mathrm{n}=1(2,5 \%)$, insulina: $\mathrm{n}=13(32,5 \%)$, sulfoniluréia + metformina: $\mathrm{n}=3(7,5 \%)$, sulfoniluréia + insulina: $\mathrm{n}=1$ $(2,5 \%)$, metformina + insulina: $n=5(12,5 \%)$, insulina + metformina + sulfoniluréia: $\mathrm{n}=1(2,5 \%)$ e nenhum: $\mathrm{n}=2(5 \%)$.

Todas foram submetidas a um exame clínico e inquérito clínico e demográfico, onde foram apurados dados relativos à idade, idade do diagnóstico da doença, tipo de tratamento utilizado, idade da menopausa e uso de terapia de reposição estrogênica, história patológica pregressa e doenças coexistentes, uso de medicamentos, índice de massa corporal (IMC), relação cintura-quadril (RCQ) e pressão arterial sistêmica.

Todas estavam na pós-menopausa, considerada pela ausência de menstruações por pelo menos um ano ou pelos níveis de gonadotrofinas plasmáticas naquelas com história de menopausa cirúrgica (FSH > 40) (20). Nenhuma delas estava em uso de reposição estrogênica ou medicações que interferissem com os hormônios sexuais.

O IMC foi calculado dividindo-se o peso $(\mathrm{kg})$ pela altura ao quadrado $\left(\mathrm{m}^{2}\right)$. Pelos resultados do IMC os indivíduos foram classificados de acordo com os critérios da Organização Mundial de Saúde (21) em 3 grupos: normal (IMC $<25 \mathrm{~g} / \mathrm{m}^{2}$ ), sobrepeso grau I (IMC $225 \mathrm{~kg} / \mathrm{m}^{2}$ e $<30 \mathrm{~kg} / \mathrm{m}^{2}$ ) e sobrepeso grau II (IMC $\geq 30 \mathrm{~kg} / \mathrm{m}^{2}$ e $<40 \mathrm{~kg} / \mathrm{m}^{2}$ ). 
Tabela 1 - Medidas antropométricas, dados demográficos e clinicos nos grupos estudados.

\begin{tabular}{llll}
\hline VARIÁVEL & $\begin{array}{l}\text { DIABÉTICAS } \\
n=40\end{array}$ & $\begin{array}{l}\text { NÃO DIABÉTICAS } \\
n=40\end{array}$ & Valor de P \\
\hline IDADE (anos) & $64,9 \pm 7,1$ & $61 \pm 8,9$ & 0,03 \\
IDADE MENOPAUSA (anos) & $49,3 \pm 0,8$ & 0,93 \\
PESO (kg) & $49 \pm 0,8$ & 0,37 \\
IMC (kg/m²) & $68,4 \pm 11,8$ & $66,4 \pm 8,5$ & 0,19 \\
OBESIDADE (sim/não) & $29,1 \pm 5,4$ & $27,7 \pm 3$ & 0,77 \\
CINTURA (cm) & $31 / 9$ & $33 / 7$ & 0,01 \\
RCQ (cintura/quadril) & $92,4 \pm 12,1$ & $86,6 \pm 9$ & 0,003 \\
Obesidade andróide sim/não-n (\%) & $0,91 \pm 0,08$ & $0,85 \pm 0,09$ & 0,03 \\
PAs (mmHg) & $30(75 \%)-10(25 \%)$ & $20(50 \%)-2(50 \%)$ & 0,01 \\
PAd (mmHg) & $155,7 \pm 20,1$ & $144,7 \pm 19,9$ & 0,63 \\
\hline
\end{tabular}

* menopausa natural: diabéticas $n=36$, não diabéticas $n=32$.

Os dados são expressos em média \pm desvio padrão ou mediana (mínimo-máximo).

A RCQ foi determinada pela divisão da circunferência abdominal (menor medida entre a cicatriz umbilical e o rebordo costal inferior) e pélvica (maior medida ao nível dos trocanteres femurais) utilizando-se fita métrica com precisão de $0,5 \mathrm{~cm}$. A distribuição de gordura foi considerada andróide ou abdominal quando a RCQ era $\geq 0,85$. Analisamos também a medida da cintura isoladamente, utilizando o valor $\geq 88 \mathrm{~cm}$ como indicativo de adiposidade abdominal (22).

Todas foram submetidas à aferição da pressão arterial (PA) em duas ocasiões, sendo considerada a média das aferições. A PA foi determinada com a paciente deitada após repouso de $5 \mathrm{~min}$, utilizando-se manguito adequado e esfignomanômetro de coluna de mercúrio padronizado e calibrado. A pressão arterial diastólica foi determinada pelo desaparecimento dos sons de Korotkoff (fase 5). A hipertensão arterial foi considerada presente quando a pressão arterial sistólica (PAs) era $\geq 140 \mathrm{mmHg} \mathrm{e} /$ ou a pressão arterial diastólica (PAd) $\geq 90 \mathrm{mmHg}$, de acordo com os critérios da Associação Americana de Cardiologia (23), ou ainda, naquelas pacientes com diagnóstico prévio de hipertensão arterial em uso de medicamentos anti-hipertensivos.

Todas foram submetidas à coleta de sangue entre 8 e $10 \mathrm{~h}$ da manhã, após jejum de $12 \mathrm{~h}$. Foram realizadas as seguintes dosagens no Laboratório Central do Hospital Universitário Pedro Ernesto: glicemia (VR: $70-110 \mathrm{mg} / \mathrm{dl}$ ), hemoglobina glicada (VR: 4,5$6,2 \%$ ), colesterol total (VR: $<220 \mathrm{mg} / \mathrm{dl}$ ), triglicerídeos (VR: $40-160 \mathrm{mg} / \mathrm{dl}$ ) e HDL colesterol (VR: $35-55 \mathrm{mg} / \mathrm{dl}$ ). O colesterol LDL foi calculado (LDLc) pela fórmula de Friedwald (24). As determinações acima foram realizadas através de reações colorimétricas, com leitura feita pelo aparelho "COBRAS-MIRA" da Roche Diagnóstica. A hemo- globina glicada foi determinada por HPLC, utilizando o aparelho L-9100 Merck Hitachi, coeficiente de variação $(\mathrm{CV})$ intraensaio e interensaio $<1 \%$, e a glicemia pelo método da glicose-oxidase.

No Laboratório de Hormônios da Disciplina de Endocrinologia foram realizadas as seguintes dosagens: peptídeo $\mathrm{C}$ - imunoensaio enzimático por quimiluminescência (DPC), com sensibilidade de $0,3 \mathrm{ng} / \mathrm{ml}, \mathrm{CV}$ intraensaio para amostras com concentrações baixas $(1,08 \pm 0,1)$ foi de $9,5 \%$ e com concentrações altas $(3,51 \pm 0,24)$ foi de $6,9 \%$; insulina RIE em fase sólida (DPC), sensibilidade de $1,2 \mu \mathrm{UI} / \mathrm{ml}, \mathrm{CV}$ intraensaio para amostras com concentrações baixas $(10,8 \pm 1,2 \mu \mathrm{UI} / \mathrm{ml})$ foi de $8,4 \% \mathrm{e}$ com concentrações altas $(112,5 \pm 9,1 \mu \mathrm{UI} / \mathrm{ml})$ foi de 8\%; estradiol - imunoensaio enzimático por quimiluminescência (DPC), sensibilidade de $10 \mathrm{pg} / \mathrm{ml}, \mathrm{CV}$ intraensaio para amostras com concentrações de $100 \pm 7,5 \mathrm{pg} / \mathrm{ml}$ foi de $7,4 \%$; testosterona - imunoensaio enzimático por quimiluminescência (DPC), sensibilidade de $100 \mathrm{pg} / \mathrm{ml}, \mathrm{CV}$ intraensaio para amostras com concentrações baixas $(571 \pm 96,4)$ foi de $17 \%$ e com concentrações altas $(3438 \pm 301,5)$ foi de $9 \%$; SHBG - imunoensaio enzimático por quimiluminescência (DPC), sensibilidade de $0,2 \mathrm{nmol} / \mathrm{l}$, $\mathrm{CV}$ intraensaio para amostras com concentrações baixas $(4,77 \pm 0,18)$ foi de $4 \%$ e com concentrações altas $(73,1 \pm 2,5)$ foi de $3 \%$. O índice de testosterona livre foi calculado dividindo-se o valor da testosterona total pelo valor da SHBG (25).

A dosagem de insulina só foi realizada em 20 pacientes diabéticas, já que o restante estava em uso de insulina exógena. Utilizamos como IRI o índice HOMA, calculado através da seguinte fórmula: insulina $(\mu \mathrm{U} / \mathrm{ml}) \times$ glicemia $(\mathrm{mmol} / \mathrm{l}) / 22,5$, para isso os 
resultados da glicemia $(\mathrm{mg} / \mathrm{dl})$ foram multiplicados por 0,05551 (26).

A análise estatística foi realizada a partir dos dados coletados de todos os pacientes e arquivados no programa de epidemiologia EPI INFO, versão 6.02, outubro de 1994, para DOS e analisados pelo programa SPSS para Windows, versão 6.0. O teste de Kolmogorov - Smirnov com nível de significância de Lilliefors foi usado para se testar a normalidade da distribuição de variáveis contínuas. Quando as variáveis não apresentavam distribuição normal utilizamos o teste de Mann-Whitney para a comparação entre duas amostras independentes. O teste de Kruskall - Wallis (H) foi utilizado para comparação de mais de duas amostras independentes, seguido do teste de MannWhitney, aplicando-se a correção de Bonferroni para comparaçōes múltiplas. Nas demais comparações utilizou-se os testes $\mathrm{t}$ de Student e a Análise de Variância. Nas tabelas de contingência utilizou-se o teste do Quiquadrado com correção de Yates ou o teste exato de Fisher. A correlação de Spearman foi utilizada quando indicada, sendo que nas variáveis que apresentaram $\mathrm{p}<0,1$, foi realizada a regressão linear univariada e posteriormente a regressão múltipla em stepwise com as variáveis significativas $(\mathrm{p}<0,05)$. Os dados são apresentados como média \pm desvio padrão (DP) ou mediana (mínimo - máximo). Considerou-se como significante um valor de $\mathrm{p}$ bicaudal $<0,05$.

\section{RESULTADOS}

As mulheres diabéticas apresentavam maior cintura $(92,4 \pm 12,1$ vs. $86,6 \pm 9 \mathrm{~cm}, \mathrm{p}=0,01)$, RCQ $(0,91 \pm 0,08$ vs. $0,85 \pm 0,09, \mathrm{p}=0,03)$, maior freqüência de distribuição andróide de gordura $(\mathrm{n}=30(75 \%)$ vs. $\mathrm{n}=20$ $(50 \%), \mathrm{p}=0,03)$, PAs $(155,7 \pm 20,1$ vs. $144,7 \pm 19,9$ $\mathrm{mmHg}, \mathrm{p}=0,01$ ) (tabela 1 ), maiores concentrações de testosterona total $(396[100-1680]$ vs. 217 [56$700] \mathrm{pg} / \mathrm{ml}, \mathrm{p}=0,003)$, índice de testosterona livre $(7,75[1,4-30,5]$ vs. $4,85[0,7-24,2], p=0,002)$ e IRI $(5[2,2-15,75]$ vs. $2,6[0,9-6,3], p=0,000)$ em comparação com as mulheres não diabéticas. Não observamos diferenças nos níveis de SHBG, estradiol, insulina e peptídeo C (tabela 2).

Não observamos diferenças nos níveis de SHBG de acordo com o IMC (tabela 3) e cintura (tabela 5). De acordo com os níveis de RCQ, observamos que as mulheres não diabéticas com distribuição andróide de gordura apresentavam menores níveis de SHBG $(44,9 \pm 18,5$ vs. $60,8 \pm 18 \mathrm{nmol} / \mathrm{l}, \mathrm{p}=0,008)$ em comparação com as mulheres não diabéticas com distribuição ginecóide (tabela 4). No grupo diabético observamos correlação entre SHBG e duração do diabetes $\left(r_{s}=0,31, p=0,04\right)$, HDL $\left(r_{s}=0,38, p=0,01\right)$, estradiol $\left(r_{s}=0,31, p=0,04\right)$, tendência com triglicerídeos $\left(r_{s}=-0,30, p=0,05\right)$ e idade $\left(r_{s}=0,26, p=0,06\right)$. No modelo de regressão múltipla em stepwise utilizando duração do DM, HDL, triglicerídeos, estradiol e idade como variáveis independentes, observamos que os triglicerídeos $\left(\mathrm{r}=-0,45, \mathrm{r}^{2}=0,20, \mathrm{p}=0,003\right)$, seguido pela idade $\left(\mathrm{r}=0,54, \mathrm{r}^{2}=0,29, \mathrm{p}=0,001\right)$ e pelo estradiol $\left(r=0,62, r^{2}=0,38, p=0,0005\right)$ se correlacionaram com a concentração de SHBG. No grupo não diabético observamos correlação entre SHBG e PAd $\left(r_{s}=-0,30, p=0,05\right), \operatorname{RCQ}\left(r_{s}=-0,39, p=0,02\right)$, glicose $\left(r_{s}=-0,48, p=0,002\right)$, índice de testosterona livre $\left(r_{s}=-0,41, p=0,008\right)$, insulina $\left(r_{s}=-0,37, p=0,01\right)$, IRI

Tabela 2 - Avaliação laboratorial nos grupos estudados.

\begin{tabular}{|c|c|c|c|}
\hline VARIÁVEL & $\begin{array}{l}\text { DIABÉTICAS } \\
\mathrm{n}=40\end{array}$ & $\begin{array}{l}\text { NÃO DIABÉTICAS } \\
\mathrm{n}=40\end{array}$ & Valor de $p$ \\
\hline $\begin{array}{l}\text { GLICOSE (mg/dl) } \\
\text { HbAlC }(\%) \\
\text { SHBG (nmol/l) } \\
\text { TESTOSTERONA }(\mathrm{pg} / \mathrm{ml}) \\
\text { ÍNDICE TESTO LIVRE } \\
\text { ESTRADIOL }(\mathrm{pg} / \mathrm{ml}) \\
\text { COLESTEROL }(\mathrm{mg} / \mathrm{dl}) \\
\text { TRIGLICERIDEOS }(\mathrm{mg} / \mathrm{dl}) \\
\text { HDL }(\mathrm{mg} / \mathrm{dl}) \\
\text { LDLC }(\mathrm{mg} / \mathrm{dl}) \\
\text { IRI } \\
\text { INSULINA }(\mathrm{m} \mid \mathrm{l} / \mathrm{ml}) \\
\text { PEPTÍDEO C }(\mathrm{ng} / \mathrm{ml})\end{array}$ & $\begin{array}{l}179,8 \pm 83,2 \\
7,6 \pm 2,1 \\
49,5 \pm 19,1 \\
396(100-1680) \\
7,75(1,4-30,5) \\
24,1 \pm 9,2 \\
248,2 \pm 52,4 \\
133,5(61-575) \\
50,9 \pm 15,6 \\
162,9 \pm 43,8 \\
5,04(2,23-15,75)^{*} \\
13,45(5,1-45,6)^{*} \\
1,5(0,3-5,6)\end{array}$ & $\begin{array}{l}89,8 \pm 11,8 \\
4,5 \pm 0,4 \\
52,8 \pm 19,7 \\
217(56-700) \\
4,85(0,7-24,2) \\
24,9 \pm 10,1 \\
228,2 \pm 38,9 \\
136,5(60-436) \\
45,6 \pm 9,5 \\
151,1 \pm 35,8 \\
2,6(0,9-6,31) \\
11,95(3,6-24,1) \\
1,7(0,5-3,1)\end{array}$ & $\begin{array}{l}0,0000 \\
0,0000 \\
0,55 \\
0,003 \\
0,002 \\
0,56 \\
0,05 \\
0,65 \\
0,08 \\
0,19 \\
0,000 \\
0,37 \\
0,56\end{array}$ \\
\hline
\end{tabular}

${ }^{*} n=20$

Os dados são expressos em média \pm desvio padrão ou mediana (mínimo-máximo). 
Tabela 3 - Comparação entre os niveis de SHBG, testosterona e índice de testosterona livre de acordo com o IMC.

\begin{tabular}{|c|c|c|c|c|c|c|}
\hline \multirow{2}{*}{$\begin{array}{l}\text { IMC } \\
\left(\mathrm{kg} / \mathrm{m}^{2}\right)\end{array}$} & \multicolumn{2}{|c|}{ SHBG (nmol/I) } & \multicolumn{2}{|c|}{ TESTOSTERONA $(\mathrm{pg} / \mathrm{ml})$} & \multicolumn{2}{|c|}{ ÍNDICE DE TESTO LIVRE } \\
\hline & DIABÉTICAS & NÃO DIABÉTICAS & DIABÉTICAS & NÄO DIABÉTICAS & DIABÉTICAS & NÄO DIABÉTICAS \\
\hline $\mathrm{IMC}<25$ & $\begin{array}{l}58,8 \pm 18,9 \\
(n=9)\end{array}$ & $\begin{array}{l}63,1 \pm 22,7 \\
(n=7)\end{array}$ & $\begin{array}{l}353,5 \pm 239 \\
(n=10)\end{array}$ & $\begin{array}{l}344 \pm 230 \\
(n=7)\end{array}$ & $\begin{array}{l}6,4 \pm 3,3 \\
(n=10)\end{array}$ & $\begin{array}{l}6.6 \pm 6.4 \\
(n=7)\end{array}$ \\
\hline $\mathrm{IMC} \geq 25$ e $<30$ & $\begin{array}{l}44,9 \pm 18,7 \\
(n=13)\end{array}$ & $\begin{array}{l}50,9 \pm 19,2 \\
(n=24)\end{array}$ & $\begin{array}{l}464 \pm 419,5 \\
(n=13)\end{array}$ & $\begin{array}{l}281.6 \pm 187.9 \\
(n=24)\end{array}$ & $\begin{array}{l}10,5 \pm 7,45 \\
(n=13)\end{array}$ & $\begin{array}{l}6,25 \pm 5,1 \\
(n=24)\end{array}$ \\
\hline $\mathrm{IMC} \geq 30$ & $\begin{array}{l}48.1 \pm 18.9 \\
(n=18)\end{array}$ & $\begin{array}{l}50,1 \pm 18,5 \\
(n=9)\end{array}$ & $\begin{array}{l}553,9 \pm 366,6^{*} \\
(n=17)\end{array}$ & $\begin{array}{l}228,4 \pm 183,5 \\
(n=9)\end{array}$ & $\begin{array}{l}13 \pm 8,9^{* *} \\
(n=17)\end{array}$ & $\begin{array}{l}5,6 \pm 6 \\
(n=9)\end{array}$ \\
\hline Valor de $p$ & 0,11 & 0,32 & 0,25 & 0,57 & 0,86 & 0,94 \\
\hline
\end{tabular}

* $p=0,01$ vs, não diabéticas

${ }^{*} \mathrm{p}=0,03$ vs. não diabéticas

Os dados são expressos em média \pm desvio padrão.

$\left(\mathrm{r}_{\mathrm{s}}=-0,43, \mathrm{p}=0,006\right)$ e tendência com peptídeo $\mathrm{C}$ $\left(r_{s}=-0,28, p=0,07\right)$. No modelo de regressão múltipla em stepwise utilizando RCQ, PAd, glicose, índice de testosterona livre, insulina, IRI e peptídeo $\mathrm{C}$ como variávêis independentes, observamos que $\mathrm{o}$ índice de testosterona livre $\left(r=-0,42, r^{2}=0,18, p=0,006\right)$, seguido do IRI $\left(r=-0,52, r^{2}=0,27, p=0,002\right)$ e da insulina $\left(\mathrm{r}=-0,62, \mathrm{r}^{2}=0,39, \mathrm{p}=0,0005\right)$ se correlacionaram com a concentração de SHBG.

Não observamos diferenças nos níveis de testosterona total de acordo com o IMC (tabela 3 ) e cintura (tabela 5). No grupo com IMC $\geq 30 \mathrm{~kg} / \mathrm{m}^{2}$, as mulheres diabéticas apresentavam maiores níveis de testosterona total do que as mulheres não diabéticas $(553,9 \pm 366,6$ vs. $228,4 \pm 183,5 \mathrm{pg} / \mathrm{ml}, \mathrm{p}=0,01)$. No grupo com cintura $\geq 88 \mathrm{~cm}$, as mulheres diabéticas apresentavam maiores níveis de testosterona do que as não diabéticas $(544,4 \pm 438,4$ vs. $265,3 \pm 202,9 \mathrm{pg} / \mathrm{ml}, \mathrm{p}=0,01)$. As mulheres não diabéticas com distribuição andróide de gordura apresentavam uma tendência a maiores níveis de testosterona $(314,5[50-700]$ vs. 184,5 [50-560] $\mathrm{pg} / \mathrm{ml}, \mathrm{p}=0,09$ ) em comparação com as mulheres não diabéticas com distribuição ginecóide (tabela 4). No grupo com distribuição ginecóide de gordura, as mulheres diabéticas apresentavam maiores níveis de testos- terona total do que as não diabéticas $(372,4 \pm 160,6$ vs. $230,5 \pm 167,3 \mathrm{pg} / \mathrm{ml}, \mathrm{p}=0,03)$. No grupo diabético observamos correlação entre testosterona total e índice de testosterona livre $\left(r_{s}=0,85, p=0,000\right)$ e tendência com IMC $\left(r_{s}=0,29, p=0,06\right)$ e estradiol $\left(r_{s}=0,29\right.$, $\mathrm{p}=0,06)$. No modelo de regressão múltipla em stepwise utilizando índice de testosterona livre, IMC e estradiol como variáveis independentes, observamos que apenas $o$ índice de testosterona livre $\left(r=0,79, r^{2}=0,62, p=0,000\right)$ se correlacionou com a concentração de testosterona total. No grupo não diabético observamos correlação entre testosterona total $\mathrm{e}$ índice de testosterona livre $\left(r_{s}=0,87, p=0,000\right)$ que se manteve na regressão univariada $\left(r=0,85, r^{2}=0,72, p=0,000\right)$.

Não observamos diferenças no índice de testosterona livre de acordo com o IMC (tabela 3 ). No grupo com IMC $\geq 30 \mathrm{~kg} / \mathrm{m}^{2}$, as mulheres diabéticas apresentavam maior índice de testosterona livre do que as não diabéticas $(13 \pm 8,9$ vs. $5,6 \pm 6, p=0,03)$. As mulheres não diabéticas com distribuição andróide de gordura apresentavam maior índice de testosterona livre $(6,4[1,1-24,3]$ vs. $3,15[0,7-9,6], p=0,01) \mathrm{em}$ comparação com as mulheres não diabéticas com distribuição ginecóide (tabela 4). No grupo com cintura $\geq 88 \mathrm{~cm}$, as mulheres diabéticas apresentavam maior

Tabela 4 - Comparação entre os níveis de SHBG, testosterona e índice de testosterona livre de acordo Com o RCQ.

\begin{tabular}{|c|c|c|c|c|c|c|}
\hline \multirow[t]{2}{*}{$\mathrm{RCQ}$} & \multicolumn{2}{|c|}{$\mathrm{SHBG}(\mathrm{nmol} / \mathrm{l})$} & \multicolumn{2}{|c|}{ TESTOSTERONA $(\mathrm{pg} / \mathrm{ml})$, } & \multicolumn{2}{|c|}{ İNDICE DE TESTO LIVRE } \\
\hline & DIABÉTICAS & NÄO DIABÉTICAS & DIABÉTICAS & NÄO DIABÉTICAS & DIABÉTICAS & NÃO DIABÉTICAS \\
\hline $\begin{array}{l}\text { Distribuição } \\
\text { andróide } \\
\text { Distribuição } \\
\text { ginecóide } \\
\text { Valor de p }\end{array}$ & $\begin{array}{l}50 \pm 18,2 \\
(n=30) \\
47,9 \pm 22,7 \\
(n=10) \\
0,75\end{array}$ & $\begin{array}{l}44,9 \pm 18,5 \\
(n=20) \\
60,8 \pm 18 \\
(n=20) \\
0,008\end{array}$ & $\begin{array}{l}515,3 \pm 403,1 \\
(n=30) \\
372,4 \pm 160,65^{*} \\
(n=10) \\
0,57\end{array}$ & $\begin{array}{l}330,7 \pm 209,4 \\
(n=20) \\
230,45 \pm 167,3 \\
(n=20) \\
0,09\end{array}$ & $\begin{array}{l}11,2 \pm 8,6 \\
(n=30) \\
8,3 \pm 3,5^{* *} \\
(n=10) \\
0,71\end{array}$ & $\begin{array}{l}8,3 \pm 6,5 \\
(n=20) \\
4,0 \pm 2,8 \\
(n=20) \\
0,01\end{array}$ \\
\hline
\end{tabular}

${ }^{*} p=0,03$ vs, não diabéticas

** $p=0,001$ vs. não diabéticas

Os dados são expressos em média \pm desvio padrão. 
Tabela 5 - Comparação entre os níveis de SHBG, testosterona e índice de testosterona livre de acordo com a cintura.

\begin{tabular}{|c|c|c|c|c|c|c|}
\hline \multirow{2}{*}{ CINTURA } & \multicolumn{2}{|c|}{ SHBG $(\mathrm{nmol} / \mathrm{l})$} & \multicolumn{2}{|c|}{ TESTOSTERONA $(\mathrm{pg} / \mathrm{ml})$} & \multicolumn{2}{|c|}{ INDDICE DE TESTO LIVRE } \\
\hline & DIABÉTICAS & NÃO DIABÉTICAS & DIABÉTICAS & NAOO DIABÉTICAS & DIABÉTICAS & NÃO DIABÉTICAS \\
\hline Cintura $\geq 88 \mathrm{~cm}$ & $\begin{array}{l}54,8 \pm 18,4 \\
(n=22)\end{array}$ & $\begin{array}{l}53,9 \pm 19,55 \\
(n=18)\end{array}$ & $\begin{array}{l}544,4 \pm 438,35^{*} \\
(n=22)\end{array}$ & $\begin{array}{l}265,3 \pm 202.9 \\
(n=18)\end{array}$ & $\begin{array}{l}12,85 \pm 9,1^{* *} \\
(n=22)\end{array}$ & $\begin{array}{l}6.1 \pm 5,7 \\
(n=18)\end{array}$ \\
\hline Cintura $<88 \mathrm{~cm}$ & $\begin{array}{l}45,1 \pm 18,9 \\
(n=18)\end{array}$ & $\begin{array}{l}51,6 \pm 20,5 \\
(n=22)\end{array}$ & $\begin{array}{l}400,4 \pm 224,8 \\
(n=18)\end{array}$ & $\begin{array}{l}293,1 \pm 189,9 \\
(n=22)\end{array}$ & $\begin{array}{l}7,72 \pm 4,4 \\
(n=18)\end{array}$ & $\begin{array}{l}6,25 \pm 5,3 \\
(n=22)\end{array}$ \\
\hline Valor de $p$ & 0.10 & 0,72 & 0.11 & 0,66 & 0,07 & 0,92 \\
\hline
\end{tabular}

$* \mathrm{p}=0,01$ vs, não diabéticas

** $\mathrm{p}=0,007$ vs. não diabéticas

Os dados são expressos em média \pm desvio padrão.

índice de testosterona livre do que as não diabéticas $(12,85 \pm 9,1$ vs. $6,1 \pm 5,7, p=0,007)$ e tendência a maior índice de testosterona livre em comparação com as diabéticas com cintura $<88 \mathrm{~cm}(12,85 \pm 9,1$ vs. $7,7 \pm 4,1$, $\mathrm{p}=0,07)$ (tabela 5). No grupo diabético observamos correlação entre índice de testosterona livre e cintura $\left(r_{s}=0,32, p=0,03\right)$, IMC $\left(r_{s}=0,38, p=0,01\right)$ e testosterona $\left(r_{s}=0,85, p=0,000\right)$. No modelo de regressão múltipla em stepwise utilizando cintura, IMC e testosterona como variáveis independentes, observamos que a testosterona $\left(\mathrm{r}=0,79, \mathrm{r}^{2}=0,62, \mathrm{p}=0,000\right)$ se correlacionou com o índice de testosterona livre. Retirando a testosterona do modelo observamos que a cintura $\left(r=0,34, r^{2}=0,12, p=0,02\right)$ também se correlacionou com índice de testosterona livre. No grupo não diabético observamos correlaçōes entre índice de testosterona livre e RCQ $\left(r_{s}=0,39, p=0,01\right)$, SHBG $\left(r_{s}=-0,41, p=0,008\right)$ e testosterona $\left(r_{s}=0,87, p=0,000\right)$. No modelo de regressão múltipla em stepwise utilizando RCQ, SHBG e testosterona como variáveis independentes, observamos que a testosterona $(r=0,85$, $\left.\mathrm{r}^{2}=0,72, \mathrm{p}=0,000\right)$, seguida pela SHBG $(\mathrm{r}=0,94$, $\left.\mathrm{r}^{2}=0,88, \mathrm{p}=0,0000\right)$, se correlacionaram com o índice de testosterona livre.

Não observamos diferenças nos níveis de estradiol de acordo com os diferentes niveis de IMC e cintura. De acordo com os níveis de RCQ, observamos que as mulheres diabéticas com distribuição andróide apresentavam maiores níveis de estradiol $(25,6 \pm 10$ vs. $17,1 \pm 7,1 \mathrm{pg} / \mathrm{ml}, \mathrm{p}=0,02$ ) em comparação com as mulheres diabéticas com distribuição ginecóide. No grupo diabético observamos uma tendência de correlação entre estradiol e SHBG $(\mathrm{r}=0,30, \mathrm{p}=0,05)$ e testosterona $(r=0,29, p=0,06)$. No modelo de regressão múltipla em stepwise observamos que a SHBG $\left(r=0,31, \mathrm{r}^{2}=0,10, \mathrm{p}=0,04\right)$ se correlacionou com a concentração de estradiol. No grupo não diabético observamos correlação entre estradiol e peptídeo $\mathrm{C}\left(\mathrm{r}_{\mathrm{s}}=0,34, \mathrm{p}=0,02\right)$ e com a cintura $\left(\mathrm{r}_{\mathrm{s}}=0,3 \mathrm{I}\right.$, $\mathrm{p}=0,04)$. No modelo de regressão múltipla não observamos nenhuma variável que se correlacionasse com o estradiol.

\section{DISCUSSĀO}

Os hormônios sexuais têm importante papel na determinação das concentrações circulantes das lipoproteínas, glicose, insulina e na distribuição regional de gordura. A observação de alterações nas concentrações plasmáticas dos esteróides sexuais e proteína carreadora em indivíduos diabéticos $(18,27)$ e suas associações com alterações no perfil lipídico e glicídico têm sido fonte para numerosos estudos $(28,29)$. Os efeitos dos esteróides sexuais sobre o metabolismo lipídico podem ser evidenciados pelas diferenças entre os sexos nas concentrações de triglicerídeos, LDL e HDL, responsáveis, pelo menos em parte, pela diferença no risco de doença cardiovascular (30). As conseqüências da administração exógena de estrogênios e androgênios sobre o metabolismo lipídico e glicídico também forneceram dados para o entendimento destas interrelações $(16,31)$.

$\mathrm{Na}$ nossa amostra, o grupo diabético apresentava maior medida da cintura e maior $\mathrm{RCQ}$, caracterizando uma maior adiposidade abdominal, confirmando os dados da literatura de maior freqüência de distribuição abdominal de gordura entre diabéticos tipo $2(27,32)$. As mulheres não diabéticas com adiposidade abdominal apresentavam maior PAs, maiores níveis de colesterol, peptídeo $\mathrm{C}$, insulina, IRI, índice de testosterona livre e menores níveis de SHBG. A associação de adiposidade abdominal com hiperinsulinemia e resistência insulínica é bem demonstrada na literatura $(33,34)$, e foi observada neste estudo, através da associação de vários indicadores da síndrome de resistência insulínica com adiposidade abdominal. A diminuição dos níveis de SHBG reflete um estado de hiperandrogenismo, já que a afinidade da SHBG pelos androgênios é bem maior que pelos 
estrogênios. Diversos estudos mostram que a obesidade visceral está associada com um estado de hiperandrogenismo entre mulheres, estando associado à resistência insulínica (33-35), fato este freqüentemente observado nas mulheres portadoras de síndrome de ovários policísticos (36) e também em mulheres com DM2 (18).

Alguns estudos epidemiológicos recentes têm sugerido que a medida isolada da cintura seria melhor preditor das alterações metabólicas associadas à adiposidade visceral do que a RCQ, sofrendo menor interferência da medida do quadril, principalmente entre as mulheres $(22,37)$. As mulheres diabéticas com cintura $\geq 88 \mathrm{~cm}$ apresentavam tendência a maior índice de testosterona livre do que aquelas com cintura $<88 \mathrm{~cm}$ e apresentavam maiores níveis de testosterona e índice de testosterona livre do que as não diabéticas. No grupo diabético observamos que a cintura explicou $12 \%$ da variabilidade do índice de testosterona livre, sugerindo que a quantidade de gordura visceral tenha importância nos níveis de androgênios nas mulheres.

No grupo com IMC $\geq 30 \mathrm{~kg} / \mathrm{m}^{2}$, as mulheres diabéticas apresentavam maiores níveis de testosterona total e índice de testosterona livre do que as não diabéticas, sugerindo que nas diabéticas o hiperandrogenismo relacionado à obesidade possa ser mais importante. Observamos correlação positiva entre IMC e testosterona e índice de testosterona livre nas mulheres diabéticas, e na análise de regressão observamos que o IMC explicou $11 \%$ da variabilidade do índice de testosterona livre, o que está de acordo com os estudos que mostram associação entre adiposidade e hiperandrogenismo, provavelmente secundária à diminuição de SHBG que acontece na obesidade (35).

As mulheres diabéticas apresentavam maiores níveis de testosterona total e índice de testosterona livre em comparação com as não diabéticas. Estudos demonstram que as mulheres diabéticas, semelhante às mulheres portadoras de ovários policísticos, comumente apresentam hiperandrogenismo associado à hiperinsulinemia $e$ adiposidade abdominal (18). Nenhuma relação causaefeito foi ainda esclarecida na literatura. A insulina é capaz de diminuir a produção e secreção hepática de SHBG (2) e estimular a produção adrenal e ovariana de androgênios (38). Por outro lado, sugere-se que o excesso de androgênios poderia causar ou agravar a resistência insulínica, por possível ação ao nível da glicogênio-sintase muscular (39). O tratamento direcionado para a perda de peso e diminuição da resistência insulínica é capaz de diminuir os níveis de androgênios e melhorar a resistência insulínica $(40)$.

Não observamos diferença significativa nos níveis de SHBG entre os grupos diabético e não dia- bético. Vários estudos comprovam que a diminuição de SHBG é um possível marcador de resistência insulínica (4I), sendo fator de risco para o desenvolvimento de diabetes tipo $2 \mathrm{em}$ mulheres e homens $(9$ 11). O fato de não termos observado menores concentrações de SHBG entre diabéticos pode ser decorrente da heterogeneidade do grupo estudado, em relação à duração da doença, grau de resistência insulínica e idade. No modelo de regressão múltipla realizado no grupo diabético observamos que os triglicerídeos, a idade e o estradiol explicaram $38 \%$ da variabilidade da concentração de SHBG. Vários estudos demonstram que com o envelhecimento ocorre aumento das concentrações de SHBG $(42,43)$, conforme sugerido pelos nossos resultados. No grupo não diabético observamos que o índice de testosterona livre, índice de resistência insulínica e insulina explicaram $39 \%$ da variabilidade da concentração de SHBG. A influência da insulinemia sobre as concentrações de SHBG é bem demonstrada na literatura $(2,4,5)$. Observamos que a SHBG explicou em torno de $10 \%$ da variabilidade do estradiol, apenas entre as mulheres diabéticas. Pasquali e cols. (44) demonstraram uma correlação entre estradiol e SHBG mais significativa, porém analisaram mulheres na prémenopausa, com maiores níveis de estrogênios, sugerindo que o estradiol, a testosterona e a insulina sejam importantes preditores da concentração de SHBG, porém em diferentes graus na pré e pós-menopausa.

As mulheres não diabéticas com distribuição abdominal de gordura apresentavam maiores níveis de estradiol do que aquelas com distribuição ginecóide. $O$ estrogênio é parcialmente responsável pela distribuição ginecóide de gordura em mulheres prémenopausa e após a menopausa haveria uma tendência a uma distribuição mais central da gordura, provavelmente por diminuição da atividade da lipase lipoproteica nos adipócitos femorais (45). A terapia de reposição estrogênica poderia reverter parcialmente esta tendência (46), embora o PEPI trial, um dos maiores estudos sobre os efeitos da terapia de reposição hormonal (TRH), não tenha demonstrado diferença na distribuição corporal de gordura em mulheres sem e com diferentes tipos de TRH (47). Certamente outros fatores relacionados ao ganho ponderal e distribuição regional de gordura, como: ingesta calórica, atividade fisica, etnia, fumo, álcool, tipo de menopausa; possam interferir na análise isolada da influência dos estrogênios sobre a composição corporal. Neste estudo as mulheres diabéticas apresentavam maior freqüência de distribuição abdominal de gordura associada com maiores níveis de androgênios, estas alterações 
poderiam explicar os maiores níveis de estradiol nestas mulheres, possivelmente por uma maior aromatização de androgênios para estrogênios no tecido adiposo.

Nas mulheres diabéticas observamos correlação negativa entre triglicerídeos e SHBG, fato este freqüentemente observado na literatura $(7,25)$. Na análise de regressão, observamos que os níveis de SHBG explicaram $20 \%$ da variabilidade da concentração de triglicerídeos. Esta influência pode ser conseqüente às concentrações tissulares de esteróides moduladas pela $S H B G$ ou secundária à presença de resistência insulínica, já que após ajuste para IRI e testosterona a correlação entre SHBG e triglicerídeos não persistiu.

Neste estudo demonstramos que as mulheres diabéticas apresentaram maior freqüência de distribuição abdominal de gordura, estando associada à maiores concentrações de testosterona total, índice de testosterona livre e estradiol e menores concentrações de SHBG. Estes dados sugerem que o hiperandrogenismo com diminuição de SHBG, freqüentemente observados entre as mulheres diabéticas com adiposidade abdominal, possam ser indicadores da Síndrome de resistência insulínica, e que poderiam, de alguma forma, agravar o grau de resistência nestes pacientes.

\section{REFERÊNCIAS}

1. Lee I, Dawson SA, Wetheral IJP, Hahnel R. SHBG secretion by human hepatocarcinoma cells is increased by both estrogens and androgens. J Clin Endocrinol Metab 1987:64:825-31.

2. Pasquali $R$, Casimirii $F$, lasio $R$. Insulin regulates testosterone and sex hormone-binding globulin concentrations in adult normal weight and obese men. J Clin Endocrinol Metab 1995;80:654-8.

3. Plymate SR, Hoop RC, Jones RE, Matej LA. Regulation of sex hormone-binding globulin production by growth factors. Metabolism 1990;39:967-70

4. Plymate SR, Matej LA, Jones RE, Friedl KL. Inhibition of SHBG in the human hepatoma (HepG2) cell line by insulin and prolactin, $J$ Clin Endocrinol Metab 1988;67:460-4.

5. Haffner S, Dunn JF, Katz MS. Relationship of sex hormone-binding globulin to lipld, lipoprotein, glucose and insulin concentrations in postmenopausal women. Metabolism 1992;41:278-84

6. Haffner S, Katz MS, Stern MP, Dunn JF. Relationship of sex hormone-binding globulin to overall adiposity and body fat distribution in a biethnic population. Int $\mathrm{J}$ Obesity 1989:13:1-9.

7. Soler JT, Folsom AR, Kaye SA, Prineas RJ. Associations of abdominal adiposity, fasting insulin, sex hormone binding globulin and estrone with lipids and lipoproteins in postmenopausal women. Atherosclerosis 1989;79:21-7.

8. Shaten J, Haffner S, Smith GD, Kuller F. Low levels of sex hormone-binding globulin and testosterone predicts the incidence of non-insulin-dependent diabetes in men. The multiple risk factor intervention trial. Diabetes 1994:43(Suppl):43A.

9. Lindstedt $G$, Lundberg $P$, Lapidus $L$, Lundgren $H$, Bengtsson $C$, Björntorp P. Low sex hormone-binding globulin concentration as independent risk factor for development of NIDDM: 12-Year follow-up of population study of women in Gothenburg, Sweden. Diabetes 1991;10:123-8

10. Haffner S, Valdez RA, Morales PA, Hazuda HP, Stern MP. Decreased sex hormone-binding globulin predicts noninsulin-dependent diabetes mellitus in women but not in men. J Clín Endocrinol Metab 1993;77:56-60.

11. Haffner S, Shaten J, Stern MP, Smith GD, Kuller L. Low levels of sex hormone-binding globulin and testosterone predict the development of non-insulin-dependent diabetes mellitus in men. Am J Epidemiol 1996; 143:889-97.

12. Evans DJ. Hoffman RG, Kalhoff RK. Relationship of androgenic activity to body fat topography, fat cell morphology and metabolic aberrations in premenopausal women. J Clin Endocrinol Metab 1983;57:304-10.

13. Andersson B, Märin P, Lissner L, Vermeulen A, Björntorp P. Testosterone concentrations in women and men with NIDDM. Diabetes Care 1994:17:405-11.

14. Haffner S, Karhapää P, Mykkänen L, Laakso M. Insulin resistance, body fat distribution and sex hormỏnes in men. Diabetes 1994:43:212-9.

15. Seidell JC, Björntorp P, Sjöström L, Kvist H, Sannerstdt R. Visceral fat accumulation in men is positively associated with insulin, glucose and C-peptide levels, but negatively with testosterone levels. Metabolism 1990;39:897-901.

16. Marin $P$, Holmang S, Johnsson L. The effects of testosterone treatment on body composition and metabolism in middle-aged men. Int J Obes 1992;16:991-7.

17. Cohen JC, Hickman R. Insulin resistance and dimished glucose tolerance in power lifters ingesting anabolic steroids. J Clin Endocrinol Metab 1987:64:960-3.

18. Polderman KH, Gooren LJG, Asscheman H, Bakker A, Heine RJ. Induction of insulin resistance by androgens and estrogens. J Clin Endocrinol Metab 1994;79:265-71.

19. National Diabetes Data Group: classification and diagnosis of diabetes mellitus and other glucose intolerance. Diabetes $1979 ; 28: 1039-57$.

20. WHO - Research on the Menopause, Report of a WHO Expert Committee. Geneva, 1981. Technical Report Series 670

21. WHO - Physical Status: The use and interpretation of anthropometry, Report of a WHO Expert Committee. Geneva, 1995 - Technical Report Series 854.

22. Han TS, et al. Waist circumference action levels in the identification of cardiovascular risk factors: prevalence study in a random sample. BMJ 1995;311:1401-5.

23. The Sixth Report of the Joint National Committee on Prevention, Detection, Evaluation and Treatment of High 
Blood Pressure. National Institute of Health Publication 98 - 4080, 1997.

24. Friedwald WT, Levy RJ, Frederickson DS. Estimation of the concentration of lowdensity lipoprotein cholesterol in plasma without use of the preparative ultracentrifuge. Clin Chem 1972; 18:499-502.

25. Stefanick ML, Willians PT, Krauss RM, Terry RB, Vranizan KM. Wood PD. Relationships of plasma estradiol, testosterone, and sex hormone-binding globulin with lipoproteins, apolipoproteins and high density lipoprotein subfractions in men. J Clin Endocrinol Metab 1987:64:723-9.

26. Haffner S, Miettinen H. Stern MP. The homeostasis model in the San Antonio heart study. Diabetes Care 1997:20:1087-92.

27. Barret-Connor E. Lower androgen levels and dyslipidemia in men with non-insulin-dependent diabetes mellitus. Ann Intern Med 1992:1 17:807-11.

28. Haffner S, Dunn JF, Katz MS. Relationship of sex hormone-binding globulin to lipid, lipoprotein, glucose and insulin concentrations in postmenopausal women. Metabolism 1992:41:278-84.

29. Handa K, Ishii H, Kono S. Behavioral correlates of plasma sex hormones and their relationships with plasma lipids and lipoproteins in Japanese men. Atherosclerosis 1997; 130:37-44.

30. Plymate SR, Swerdloff RS. Androgen, lipids, and cardiovascular risk. Ann Int Med 1992;117:871-2.

31. Crook D. Postmenopausal hormone replacement therapy, lipoprotein metabolism and coronary heart disease. J Cardiovasc Pharm 1996;28(suppl 5):\$40-5.

32. Cowie CC. Howard BV, Harris MI. Serum lipoproteins in African Americans and whites with non-insulin-dependent diabetes in the US population. Circulation 1994;90: $1185-93$

33. Soler JT, Folsom AR, Kaye SA, Prineas RJ. Associations of abdominal adiposity, fasting insulin, sex hormone binding globulin and estrone with lipids and lipoproteins in postmenopausal women. Atherosclerosis 1989:79:21-7.

34. Björntorp P. Metabolic implications of body fat distribution. Diabetes Care 1991;14:1132-43.

35. Haffner S, Katz MS, Dunn JF. Increased upper body and overall adiposity is associated with decreased sex hormone binding globulin in postmenopausal women. Int $\mathrm{J}$ Obesity 1991;15:471-8.

36. Pasquali R, Casimirri F, Lobate AM. Insulin and androgen relationships with abdominal body fat distribution in women with and without hyperandrogenism. Horm Res 1993:39:179-87.

37. Pouliot MC. Després JP, Lemieux S. Waist circumference and abdominal sagital diameter: best simple anthropometric tissue accumulation and related cardiovascular risk in men and women. Am J Card 1994:73:460-8.

38. Poretsky L. On the paradox of insulin-induced hyperandrogenism in insulin-resistant states. Endocr Rev $1991: 12: 3-13$

39. Holmang A, Svedberg J, Jennische E, Bjorntörp P. Effects of testosterone on muscle insulin sensitivity and morphology in female rats. Am J Physiol 1990;259:E555-60.

40. Pasquali $R$, Vicennati $V$, Bertazzo D. Clinical and hormonal characteristics of obese amenorrheic hyperandrogenic women before and after weight loss. J Clin Endocrinol Metab 1989;68:173-9.

41. Nestler JE. Editorial: Sex hormone-binding globulin: a marker for hyperinsulinemia and/or insulin resistance? J Clin Endocrinol Metab 1993;76:273-4.

42. Field AE, Colditz GA, Willett WC, Longcope C, MCKinlay $\mathrm{JB}$. The relation of smoking, age, relative weight and dietary intake to serum adrenal steroids, sex hormones, and sex hormone-binding globulin in middle-aged men. J Clin Endocrinol Metab 1994:79:1310-6.

43. Goodman-Gruen D, Barret-Connor E. A prospective study of sex hormone-binding globulin and fatal cardiovascular disease in Rancho Bernardo men and women. $J$ Clin Endocrinol Metab 1996;81:2999-3003.

44. Pasquali R, Vicennati $V$, Bertazzo D. Determinants of sex hormone-binding globulin blood concentrations in premenopausal and postmenopausal women with different estrogen status. Metabolism 1997:46:5-9.

45. Rebuffé-Scrive M, Lönnroth $P$. Marin $P$. Regional adipose tissue metabolism in men and postmenopausal women. Int J Obes 1987: 11:347-55.

46. Haarbo J, Marslew U, Gotfresdsen A, Chistiansen C. Postmenopausal hormone replacement prevents central distribution of body fat after menopause. Metabolism 1991:40:1323-6.

47. The Writing Group for the Pepi Trial. Effects of estrogen or estrogen/progestin regimens on heart disease risk factors in postmenopausal women: The postmenopausal estrogen/progestin interventions (PEPI) trial. JAMA $1995 ; 273: 199-208$

\section{Endereço para correspondência:}

Luciana Bahia

Av. Visconde de Albuquerque 986/102

22450-000 Rio de Janeiro, RJ 